\title{
Article \\ New Study of the Existence and Dimension of the Set of Solutions for Nonlocal Impulsive Differential Inclusions with a Sectorial Operator
}

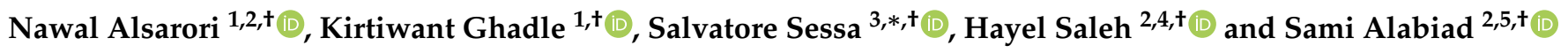 \\ 1 Department of Mathematics, Dr. Babasaheb Ambedkar Marathwada University, Aurangabad 431004, India; \\ n_alsarori@yahoo.com (N.A.); ghadle.maths@bamu.ac.in (K.G.) \\ 2 Department of Mathematics, Taiz University, Taiz 6803, Yemen; hnsaleh112@myamu.ac.in (H.S.); \\ ssaif1@ksu.edu.sa (S.A.) \\ 3 Dipartimento di Architettura, Università degli Studi di Napoli Federico II, Via Toledo 402, 80134 Napoli, Italy \\ 4 Department of Mathematics, Aligarh Muslim University, Aligarh 202002, India \\ 5 Department of Mathematics, King Saud University, Riyadh 11451, Saudi Arabia \\ * Correspondence: sessa@unina.it \\ + These authors contributed equally to this work.
}

Citation: Alsarori, N.; Ghadle, K.; Sessa, S.; Saleh, H.; Alabiad, S. New Study of Existence and Dimension of the Set of Solutions for Nonlocal Impulsive Differential Inclusions with Sectorial Operator. Symmetry 2021, 13, 491. https://doi.org/10.3390/ sym13030491

Academic Editors: Wei-Shih Du, Alicia Cordero Barbero, Huaping Huang and Juan Ramón Torregrosa Sánchez

Received: 20 February 2021

Accepted: 12 March 2021

Published: 17 March 2021

Publisher's Note: MDPI stays neutra with regard to jurisdictional claims in published maps and institutional affiliations.

Copyright: (c) 2020 by the authors. Licensee MDPI, Basel, Switzerland. This article is an open access article distributed under the terms and conditions of the Creative Commons Attribution (CC BY) license (https:/ / creativecommons.org/licenses/by/ $4.0 /)$.
Abstract: In this article, we are interested in a new generic class of nonlocal fractional impulsive differential inclusions with linear sectorial operator and Lipschitz multivalued function in the setting of finite dimensional Banach spaces. By modifying the definition of PC-mild solutions initiated by Shu, we succeeded to determine new conditions that sufficiently guarantee the existence of the solutions. The results are obtained by combining techniques of fractional calculus and the fixed point theorem for contraction maps. We also characterize the topological structure of the set of solutions. Finally, we provide a demonstration to address the applicability of our theoretical results.

Keywords: impulsive fractional differential inclusions; nonlocal conditions; fixed point theorems; mild solutions

\section{Introduction}

Fractional calculus deals with integrations and derivatives in case of a non-integer order, which is a generalized shape of classical integrals and derivatives. The significance of fractional calculus is due to its numerous applications in many significant scientific fields such as physics, control theory, fluid dynamics, image processing, computer networking, sign processing, biology and others. During the previous couple of decades, fractional calculus has been substantially studied and there are many researchers who have demonstrated a deep interest in it [1-8].

On the other hand, there are numerous examples in biotechnology, physics, population dynamics and processes economics which are characterized by the reality that the model parameters are subjected to short-time period perturbations. For instance, in treating some diseases, impulses can correspond to the management of drug treatment; in environmental sciences, impulses can correspond to seasonal modifications or harvesting; in economics, impulses can additionally correspond with sudden prices adjustments. Adequate tools to remedy such techniques and phenomena are impulsive differential equations and inclusions. The first ones had been extensively researched in finite and Banach spaces; see, for instance, the studies [9-13]. Recently, the existence of mild solutions for the impulsive differential equations and inclusions in Banach spaces have been examined through many researchers, we refer the reader to [14-24]. 
In this paper, we are interested in the existence of mild solutions to the following nonlocal impulsive fractional differential inclusions of the type

$$
\begin{cases}{ }^{c} D_{t}^{\alpha} x(t) \in A x(t)+F(t, x(t)), & t \in J=[0, b], t \neq t_{i} \\ \Delta x\left(t_{i}\right)=I_{i}\left(x\left(t_{i}^{-}\right)\right), & i=1, \ldots, m \\ x(0)+g(x)=x_{0}, & x_{0} \in \mathbb{R}^{n}\end{cases}
$$

where ${ }^{c} D^{\alpha}, 0<\alpha<1$, is the Caputo derivative, $A$ is sectorial operator on $\mathbb{R}^{n}, F: J \times \mathbb{R}^{n} \rightarrow$ $P\left(\mathbb{R}^{n}\right)$ is a multifunction, $0=t_{0}<t_{1}<\cdots<t_{m}<t_{m+1}=b, I_{i}: \mathbb{R}^{n} \rightarrow \mathbb{R}^{n}, 1 \leq i \leq m$, are impulsive functions, $\Delta x\left(t_{i}\right)=x\left(t_{i}^{+}\right)-x\left(t_{i}^{-}\right), x\left(t_{i}^{-}\right)=\lim _{s \rightarrow t_{i}^{-}} x(s), x\left(t_{i}^{+}\right)=\lim _{s \rightarrow t_{i}^{+}} x(s)$ and $g: P C\left(J, \mathbb{R}^{n}\right) \rightarrow \mathbb{R}^{n}$.

It is worth mentioning that the topic of evolution inclusions and equations involving sectorial or almost sectorial terms has been extensively examined (see [25-33]).

Shu et al. [29] gave a new and different concept of mild solutions for the following equation:

$$
\begin{cases}{ }^{c} D^{q} x(t)=A x(t)+f(t, x(t)), & t \in J=[0, b], t \neq t_{i}, \\ \Delta x\left(t_{i}\right)=I_{i}\left(x\left(t_{i}^{-}\right)\right), & i=1, \cdots, m, \\ x(0)=x_{0}, & \end{cases}
$$

where $f$ is a completely continuous single function and $A$ is a sectorial operator with the operators families $\left\{\mathcal{S}_{\alpha}(t): t \geq 0\right\}$ and $\left\{\mathcal{T}_{\alpha}: t \geq 0\right\}$ are compact.

Agarwal et al. [25] used the definition of mild solution given by Shu in [29] to prove an existence result for the inclusion:

$$
\left\{\begin{array}{l}
{ }^{c} D^{q} x(t) \in A x(t)+f(t, x(t)), \quad t \in J=[0, b], t \neq t_{k}, \\
x(0)+h(x)=x_{0} \in \mathbb{R}^{n}
\end{array}\right.
$$

where the linear part is fractional sectorial. They also studied the dimension of the set of mild solutions.

Shu et al. [30] proved that the solutions obtained in [29] are not correct and presented the right form of the solutions for (2) when $0<\alpha<1$ and $1<\alpha<2$.

Motivated by the above-mentioned results, we propose to study the problem (1); we first introduce the modified form of solutions for (1) based on the definition given by Shu [30]. Second, we use our formula to extend the results obtained in [25] to nonlocal fractional inclusions associated with impulses effect. Moreover, we study the topological structure of the set of solutions.

\section{Preliminaries}

In this part, we present some primary concepts, definitions and initial facts which are useful for the development of this article.

Let $C\left(J, \mathbb{R}^{n}\right)=\left\{\mu: J \rightarrow \mathbb{R}^{n}: \mu\right.$ is continuous $\}$, with the norm $\|x\|=\sup \{\|x(t)\|: t \in J\}$, $L^{1}\left(J, \mathbb{R}^{n}\right)=\left\{\mathcal{G}: J \rightarrow \mathbb{R}^{n}: \mathcal{G}\right.$ is Bochner integrable $\}, \mathcal{L}\left(\mathbb{R}^{n}\right)$ is the Banach space of all linear operators bounded on $\mathbb{R}^{n}$. Let $(E, d)$ be a metric space, we denote:

$$
\begin{aligned}
P_{b}(E) & =\{X: X \subset E, X \neq \varnothing, X \text { is bounded }\}, \\
P_{c l}(E) & =\{X: X \subset E, X \neq \varnothing, X \text { is closed }\}, \\
P_{k}(E) & =\{X: X \subset E, X \neq \varnothing, X \text { is compact }\}, \\
P_{c}(E) & =\{X: X \subset E, X \neq \varnothing, X \text { is convex }\}, \\
P_{c k}(E) & =\{X: X \subset E, X \neq \varnothing, X \text { is convex and compact }\} .
\end{aligned}
$$


Let $H_{d}: P(E) \times P(E) \rightarrow \mathbb{R} \cup\{\infty\}$ define by

$$
H_{d}(X, Y)=\max \left\{\sup _{x \in X} d(x, Y), \sup _{y \in Y} d(y, X)\right\},
$$

where $d(x, Y)=\inf _{y \in Y} d(x, y)$ and $d(y, X)=\inf _{x \in X} d(x, y) . H_{d}$ is the Pompeiu Hausdorff functional. It is well known that $\left(P_{c l, b}(E), H_{d}\right)$ is a metric space and $\left(P_{c l}(E), H_{d}\right)$ is a generalized metric space. For more details see [34].

Let $\left.\left.J_{0}=\left[0, t_{1}\right], J_{i}=\right] t_{i}, t_{i+1}\right], i=1, \cdots, m$, and

$$
P C\left(J, \mathbb{R}^{n}\right)=\left\{x: J \rightarrow \mathbb{R}^{n}, x_{\left.\right|_{i}} \in C\left(J_{i}, \mathbb{R}^{n}\right) \text {, with } x\left(t_{i}^{+}\right) \text {and } x\left(t_{i}^{-}\right) \text {exist }\right\} .
$$

The space $P C\left(J, \mathbb{R}^{n}\right)$ is a Banach space with the norm

$$
\|x\|_{P C}=\max \{\|x(t)\|: t \in J\} .
$$

Now, let us recall some important definitions and lemmas on multivalued functions and fractional calculus.

Definition 1. ([35], [36]).

Let $X$ and $Y$ be two topological spaces. A multifunction $F: X \rightarrow P(Y)$ is said to be:

1. Upper semi-continuous (u.s.c) if $F^{-1}(W)=\{x \in X: F(x) \subseteq W\}$ is open for every open subset $W$ of $Y$.

2. Lower semi-continuous (l.s.c) if $F^{+1}(W)=\{x \in X: F(x) \cap W \neq \varnothing\}$ is open for every open subset $W$ of $Y$.

3. Continuous if it is both (u.s.c) and (l.s.c).

4. Completely continuous if $F(V)$ is relatively compact for every bounded subset $V$ of $X$.

Definition 2. Let $F: X \rightarrow P(Y)$. A multifunction $F$ is said to has a fixed point $x$, if $x \in F(x)$, $x \in X$. The fixed points set of $F$ is denoted by Fix $(F)$.

Definition 3. A sequence $\left\{f_{n}: n \in \mathbb{N}\right\} \subset L^{1}(J, E)$ is called semi-compact if:

1. It is integrably bounded i.e. there is $q \in L^{1}\left(J, \mathbb{R}^{+}\right)$such that $\left\|f_{n}(t)\right\| \leq q(t)$ a.e. $t \in J$.

2. The set $\left\{f_{n}(t): n \in \mathbb{N}\right\}$ is relatively compact in $E$ a.e. $t \in J$.

Lemma 1. ([36]). Every semi-compact sequence in $L^{1}(J, E)$ is weakly compact.

Definition 4. The multivalued map $F: E \rightarrow P_{c l}(E)$ is called

(i) $\eta$-Lipschitz if there is $\eta>0$ with

$$
H_{d}(F(x), F(y)) \leq \eta d(x, y), \quad x, y \in E .
$$

(ii) $\eta$-contraction if it is $\eta$-Lipschitz with $\eta<1$.

Lemma 2. Let $F: E \rightarrow P_{k}(E)$ be $\eta$-contraction. Then $F$ is continuous with respect to Hausdorff metric.

Definition 5. Let $F: J \rightarrow P_{c l}\left(\mathbb{R}^{n}\right)$. If the function $t \rightarrow d(x, F(t))=\inf \{\|x-y\|: y \in F(t)\}$ is measurable for all $x \in \mathbb{R}^{n}$, then $F$ is measurable.

Lemma 3. ([37]). Let $F: J \rightarrow P_{c k}\left(\mathbb{R}^{n}\right)$ be a measurable function with $\chi\{t: \operatorname{dim} F(t)<1\}=0$, where $\chi$ is the Lebesgue measure. Then there are arbitrarily many linearly independent measurable selections $f_{1}(\cdot), f_{2}(\cdot), \cdots, f_{m}(\cdot)$ of $F$. 
Lemma 4. ([37]). Let $B$ be a nonempty, convex and closed subset of a Banach space X. Suppose that $Q: B \rightarrow P_{c k}(B)$ is $\eta$-contraction. If $f: B \rightarrow B$ is a continuous selection of $Q$, then Fix $(f) \neq \varnothing$.

Lemma 5. (Saint Raymond [38]). Let $X$ be a Banach space, $Y$ a compact metric space with dim $Y<n$ and $Q: Y \rightarrow P_{c k}(X)$ a lower semi-continuous map with $0 \in Q(x)$ and $\operatorname{dim} Q(x) \geq n$ for every $x \in Y$. Then, there exists a continuous selection $g$ of $Q$ such that $g(x) \neq 0$ for each $x \in Y$.

Lemma 6. (Michael's selection Theorem, [39]). Let $X$ be a Banach space, $Y$ a metric space and $Q: Y \rightarrow P_{c, c l}(X)$ a lower semi-continuous map. Then, there exists a continuous selection $f: Y \rightarrow X$ of $Q$.

Definition 6. ([4]). The Riemann-Liouville fractional integral of order $\alpha>0$ of a continuous function $f$ is defined by

$$
I^{\alpha} f(t)=\frac{1}{\Gamma(\alpha)} \int_{0}^{t}(t-s)^{\alpha-1} f(s) d s, \quad t>0,
$$

provided that the right-hand side is point-wise defined on $[0, \infty)$.

Definition 7. ([4]). Let $f \in C^{n}([0, \infty), \mathbb{R})$. The Caputo derivative of fractional order $\alpha>0$ for $f$ is defined by

$$
\begin{aligned}
{ }^{c} D^{\alpha} f(t) & =\frac{1}{\Gamma(n-\alpha)} \int_{0}^{t}(t-s)^{n-\alpha-1} f^{n}(s) d s \\
& =I^{n-\alpha} f^{n}(t), n-1<\alpha<n, \quad n=[\alpha]+1,
\end{aligned}
$$

where $[\alpha]$ is the integer part of the real number $\alpha$.

For further details on fractional calculus, we refer to [4-6].

Definition 8. Let $A: D(A) \subset \mathbb{R}^{n} \rightarrow \mathbb{R}^{n}$ be a linear closed operator. $A$ is said to be sectorial of type $(M, \theta, \omega)$ if $\exists \omega \in \mathbb{R}, \theta \in\left(0, \frac{\pi}{2}\right)$ and $M>0$ such that the resolvent of $A$ exists outside the sector

$$
\omega+S_{\theta}=\{\omega+\lambda: \lambda \in \mathbb{C},|\operatorname{Arg}(-\lambda)|<\theta\}
$$

and

$$
\left\|(\lambda I-A)^{-1}\right\| \leq \frac{M}{|\lambda-\omega|}, \lambda \notin \omega+S_{\theta} .
$$

Lemma 7. ([30]). Assume that $A$ is sectorial of type $(M, \theta, \omega)$. If $0<\alpha<1$, then

(i) ${ }^{c} D^{\alpha}\left(\mathcal{T}_{\alpha}(t) x_{0}\right)=A\left(\mathcal{T}_{\alpha}(t) x_{0}\right)$.

(ii) ${ }^{c} D^{\alpha}\left(\int_{0}^{t} \mathcal{S}_{\alpha}(t-s) f(s) d s\right)=A \int_{0}^{t} \mathcal{S}_{\alpha}(t-s) f(s) d s+f(t)$,

where $\mathcal{T}_{\alpha}(t)=\frac{1}{2 \pi i} \int_{Y} e^{\lambda t} \lambda^{\alpha-1} R\left(\lambda^{\alpha}, A\right) d \lambda, \quad \mathcal{S}_{\alpha}(t)=\frac{1}{2 \pi i} \int_{Y} e^{\lambda t} R\left(\lambda^{\alpha}, A\right) d \lambda, Y$ is a suitable path such that $\lambda^{\alpha} \notin \omega+S_{\theta}$ for $\lambda \in \mathrm{Y}$ and $R\left(\lambda^{\alpha}, A\right)=\left(\lambda^{\alpha} I-A\right)^{-1}$.

Lemma 8. ([29]). Let $\alpha \in(0,1)$ and $A \in A^{\alpha}\left(\theta_{0}, \omega_{0}\right)$. Then for all $t>0$ we have $\left\|\mathcal{T}_{\alpha}(t)\right\|_{\mathcal{L}\left(\mathbb{R}^{n}\right)} \leq M e^{\omega t}, \quad\left\|\mathcal{S}_{\alpha}(t)\right\|_{\mathcal{L}\left(\mathbb{R}^{n}\right)} \leq C e^{\omega t}\left(1+t^{\alpha-1}\right), \quad \omega>\omega_{0}$. Let

$$
M_{\mathcal{T}_{\alpha}}=\sup _{0 \leq t \leq b}\left\|\mathcal{T}_{\alpha}(t)\right\|_{\mathcal{L}\left(\mathbb{R}^{n}\right)}, M_{\mathcal{S}_{\alpha}}=\sup _{0 \leq t \leq b} C e^{\omega t}\left(1+t^{\alpha-1}\right)
$$

Then,

$$
\left\|\mathcal{T}_{\alpha}(t)\right\|_{\mathcal{L}\left(\mathbb{R}^{n}\right)} \leq M_{\mathcal{T}_{\alpha}},\left\|\mathcal{S}_{\alpha}(t)\right\|_{\mathcal{L}\left(\mathbb{R}^{n}\right)} \leq t^{\alpha-1} M_{\mathcal{S}_{\alpha}}
$$

For more information about the sectorial operator we refer the reader to [29]. 
Theorem 1. (Covitz and Nadler [40]). Let $(E, d)$ be a complete metric space. If $Q: E \rightarrow P_{c l}(E)$ is $\eta$-contraction, then $\operatorname{Fix}(Q) \neq \varnothing$.

\section{Main Results}

Lemma 9. Let

$$
\left(P_{1}\right)\left\{\begin{array}{l}
{ }^{c} D_{t}^{\alpha} x(t)=A x(t)+f(t), \quad t \in[0, b], t \neq t_{i}, 0<\alpha<1, \\
\Delta x\left(t_{i}\right)=I_{i}\left(x\left(t_{i}^{-}\right)\right), \\
x(0)=x_{0}-g(x),
\end{array}\right.
$$

where $f:[0, b] \rightarrow \mathbb{R}^{n}$. If $f$ satisfies the uniform Hölder condition with exponent $\delta \in(0,1]$ and $A$ is sectorial operator of type $(M, \theta, \omega)$, then $\left(P_{1}\right)$ has unique solution $x(t) \in P C\left(J, \mathbb{R}^{n}\right)$ defined as:

$$
x(t)= \begin{cases}\mathcal{T}_{\alpha}(t)\left(x_{0}-g(x)\right)+\int_{0}^{t} \mathcal{S}_{\alpha}(t-s) f(s) d s, & t \in J_{0}, \\ \mathcal{T}_{\alpha}(t)\left(x_{0}-g(x)\right)+\sum_{k=1}^{k=i} \mathcal{T}_{\alpha}\left(t-t_{k}\right) I_{k}\left(x\left(t_{k}^{-}\right)\right) & \\ +\int_{0}^{t} \mathcal{S}_{\alpha}(t-s) f(s) d s, & t \in J_{i}, 1 \leq i \leq m .\end{cases}
$$

Proof. In order to show that $x(t)$ is a solution of $\left(P_{1}\right)$, we consider the following two cases: Case I. If $t \in\left[0, t_{1}\right]$, we have

$$
x(t)=\mathcal{T}_{\alpha}(t)\left(x_{0}-g(x)\right)+\int_{0}^{t} \mathcal{S}_{\alpha}(t-s) f(s) d s
$$

Case II. If $\left.\left.t \in J_{i}=\right] t_{i}, t_{i+1}\right]$, we have

$$
x(t)=\mathcal{T}_{\alpha}(t)\left(x_{0}-g(x)\right)+\sum_{k=1}^{k=i} \mathcal{T}_{\alpha}\left(t-t_{k}\right) I_{k}\left(x\left(t_{k}^{-}\right)\right)+\int_{0}^{t} \mathcal{S}_{\alpha}(t-s) f(s) d s .
$$

By taking Caputo derivative to both sides of (5) and making use of Lemma 7, we get

$$
\begin{aligned}
{ }^{c} D^{\alpha} x(t) & ={ }^{c} D^{\alpha}\left[\mathcal{T}_{\alpha}(t)\left(x_{0}-g(x)\right)+\sum_{k=1}^{k=i} \mathcal{T}_{\alpha}\left(t-t_{k}\right) I_{k}\left(x\left(t_{k}^{-}\right)\right)+\int_{0}^{t} \mathcal{S}_{\alpha}(t-s) f(s) d s\right] \\
& =A \mathcal{T}_{\alpha}(t)\left(x_{0}-g(x)\right)+A \sum_{k=1}^{k=i} \mathcal{T}_{\alpha}\left(t-t_{k}\right) I_{k}\left(x\left(t_{k}^{-}\right)\right)+A \int_{0}^{t} \mathcal{S}_{\alpha}(t-s) f(s) d s+f(t) \\
& =A x(t)+f(t) .
\end{aligned}
$$

when $t=0$, we have

$$
x(0)=\mathcal{T}_{\alpha}(0)\left(x_{0}-g(x)\right)+\int_{0}^{0} \mathcal{S}_{\alpha}(0-s) f(s)=x_{0}-g(x) .
$$

Furthermore,

$$
\begin{aligned}
\Delta x\left(t_{i}\right) & =x\left(t_{i}^{+}\right)-x\left(t_{i}^{-}\right) \\
& =\sum_{k=1}^{k=i} \mathcal{T}_{\alpha}\left(t-t_{k}\right) I_{k}\left(x\left(t_{k}^{-}\right)\right)-\sum_{k=1}^{k=(i-1)} \mathcal{T}_{\alpha}\left(t-t_{k}\right) I_{k}\left(x\left(t_{k}^{-}\right)\right) \\
& =\mathcal{T}_{\alpha}\left(t_{i}-t_{i}\right) I_{i}\left(x\left(t_{i}^{-}\right)\right) \\
& =\mathcal{T}_{\alpha}(0) I_{i}\left(x\left(t_{i}^{-}\right)\right) \\
& =I_{i}\left(x\left(t_{i}^{-}\right)\right) .
\end{aligned}
$$

This shows that $(4)$ is solution for $\left(P_{1}\right)$.

Now, we will give the definition of a mild solution for (1). 
Definition 9. Let $A$ be a sectorial of type $(M, \theta, \omega)$ and $\alpha \in(0,1)$. A function $x \in P C\left(J, \mathbb{R}^{n}\right)$ is a mild solution for (1) if

$$
x(t)= \begin{cases}\mathcal{T}_{\alpha}(t)\left(x_{0}-g(x)\right)+\int_{0}^{t} \mathcal{S}_{\alpha}(t-s) f(s) d s, & t \in J_{0}, \\ \mathcal{T}_{\alpha}(t)\left(x_{0}-g(x)\right)+\sum_{k=1}^{k=i} \mathcal{T}_{\alpha}\left(t-t_{k}\right) I_{k}\left(x\left(t_{k}^{-}\right)\right) & \\ +\int_{0}^{t} \mathcal{S}_{\alpha}(t-s) f(s) d s, & t \in J_{i}, 1 \leq i \leq m,\end{cases}
$$

where $f \in S_{F(t, x(t))}^{1}=\left\{f \in L^{1}\left(J, \mathbb{R}^{n}\right), f(t) \in F(t, x(t))\right.$ for a.e. $\left.t \in J\right\}$.

We will show our result for (1) by using the following conditions:

$H_{1}: A$ is a sectorial operator of type $(M, \theta, \omega)$.

$H_{2}$ : Let $F: J \times \mathbb{R}^{n} \rightarrow P_{c k}\left(\mathbb{R}^{n}\right)$, such that for every $x \in \mathbb{R}^{n}, t \rightarrow F(t, x)$ is measurable.

$H_{3}$ : There is a function $\vartheta \in C\left(J, \mathbb{R}^{+}\right)$with

(a) $\quad H_{d}(F(t, x), F(t, y)) \leq \vartheta(t)\|x-y\|$, for a.e. $t \in J, x, y \in \mathbb{R}^{n}$.

(b) $\quad\|F(t, x)\|=\sup \{\|u\|: u \in F(t, x)\} \leq \vartheta(t)$, a.e. $t \in J$ and $x \in \mathbb{R}^{n}$.

$H_{4} \quad: g: P C\left(J, \mathbb{R}^{n}\right) \rightarrow \mathbb{R}^{n}$ is continuous and there exists a constant $N>0$ with

$$
\|g(x)-g(y)\| \leq N\|x-y\|, \forall x, y \in P C\left(J, \mathbb{R}^{n}\right) .
$$

$H_{5} \quad$ : For every $i=1, \cdots, m, I_{i}: \mathbb{R}^{n} \rightarrow \mathbb{R}^{n}$ is continuous and there exists a constant $\zeta_{i}>0$ with

$$
\left\|I_{i}(x)-I_{i}(y)\right\| \leq \zeta_{i}\|x-y\|, \quad \forall x, y \in \mathbb{R}^{n} .
$$

Theorem 2. Suppose that $H_{1}-H_{5}$ hold. Then the problem (1) has a solution on J provided that

$$
M_{\mathcal{T}_{\alpha}}(N+\zeta)+M_{\mathcal{S}_{\alpha}} \frac{b^{\alpha}}{\alpha}\|\vartheta\|<1
$$

where $\zeta=\sum_{i=1}^{m} \zeta_{i}$.

Proof. For every $x \in P C\left(J, \mathbb{R}^{n}\right)$, let

$$
S_{F(\cdot, x(\cdot))}^{1}=\left\{f \in L^{1}\left(J, \mathbb{R}^{n}\right): f(t) \in F(t, x(t)) \text { for a.e. } t \in J\right\} .
$$

By using $H_{2}, H_{3}$ and Theorem III.6 in [41], we get $F(t, x(t))$ is measurable. Moreover, $F(t, x(t))$ has measurable selection $f(t)$ and from the condition $H_{3}$ part (b) we have $\|f(t)\| \leq\|F(t, x(t))\| \leq \vartheta(t)$. Then, $S_{F(\cdot, x(\cdot))}^{1} \neq \varnothing \forall x \in P C\left(J, \mathbb{R}^{n}\right)$. Now, let $Q$ : $P C\left(J, \mathbb{R}^{n}\right) \rightarrow 2^{P C\left(J, \mathbb{R}^{n}\right)}$ defined as $y \in Q(x)$ iff:

$$
y(t)= \begin{cases}\mathcal{T}_{\alpha}(t)\left(x_{0}-g(x)\right)+\int_{0}^{t} \mathcal{S}_{\alpha}(t-s) f(s) d s, & t \in J_{0}, \\ \mathcal{T}_{\alpha}(t)\left(x_{0}-g(x)\right)+\sum_{k=1}^{k=i} \mathcal{T}_{\alpha}\left(t-t_{k}\right) I_{k}\left(x\left(t_{k}^{-}\right)\right) & \\ +\int_{0}^{t} \mathcal{S}_{\alpha}(t-s) f(s) d s, & t \in J_{i}, 1 \leq i \leq m .\end{cases}
$$

It is simple to prove that any fixed point for $Q$ is mild solution for (1). So, we will prove that $Q$ satisfies the assumptions of Theorem 1. As the first step, we prove that $Q(x)$ is closed for every $x \in P C\left(J, \mathbb{R}^{n}\right)$. Let $\left\{w_{n}\right\}_{n \geq 1}$ be a sequence in $Q(x)$ such that $w_{n} \rightarrow w$ in $P C\left(J, \mathbb{R}^{n}\right)$. Our goal is to show that $w \in Q(x)$. As $\left\{w_{n}\right\}_{n \geq 1} \in Q(x)$, there is $\left\{f_{n}\right\}_{n \geq 1}$ in $S_{F(\cdot, x(\cdot))}^{1}$ with

$$
w_{n}(t)= \begin{cases}\mathcal{T}_{\alpha}(t)\left(x_{0}-g(x)\right)+\int_{0}^{t} \mathcal{S}_{\alpha}(t-s) f_{n}(s) d s, & t \in J_{0}, \\ \mathcal{T}_{\alpha}(t)\left(x_{0}-g(x)\right)+\sum_{k=1}^{k=i} \mathcal{T}_{\alpha}\left(t-t_{k}\right) I_{k}\left(x\left(t_{k}^{-}\right)\right) & \\ +\int_{0}^{t} \mathcal{S}_{\alpha}(t-s) f_{n}(s) d s, & t \in J_{i}, 1 \leq i \leq m .\end{cases}
$$


By $\left(H_{3}\right)$ we have for every $n \geq 1$ and a.e. $t \in J,\left\|f_{n}(t)\right\| \leq \vartheta(t)$. So, $\left\{f_{n}: n \geq 1\right\}$ is integrable bounded. Moreover, because $\left\{f_{n}(t): n \geq 1\right\} \subset F(t, x(t))$, for a.e. $t \in J$, the set $\left\{f_{n}(t): n \geq 1\right\}$ is relatively compact in $\mathbb{R}^{n}$ for a.e. $t \in J$. Therefore, the set $\left\{f_{n}: n \geq 1\right\}$ semicompact. By Lemma 1 , it is weakly compact in $L^{1}\left(J, \mathbb{R}^{n}\right)$. We may assume that $\left(f_{n}\right)_{n \geq 1}$ converges weakly to $f \in L^{1}\left(J, \mathbb{R}^{n}\right)$. By Mazur's Lemma there exists a sequence $\left\{\mathcal{V}_{n}\right\}_{n=1}^{\infty} \subseteq \overline{\operatorname{conv}}\left\{f_{n}: n \geq 1\right\}$ such that $\mathcal{V}_{n}$ converges strongly to $f$. Since $F$ has convex and compact values, the set $S_{F(\cdot, x(\cdot))}^{1}$ is convex and compact. Therefore, $\left\{\mathcal{V}_{n}\right\}_{n=1}^{\infty} \subseteq S_{F(\cdot, x(\cdot))}^{1}$ and $f \in S_{F(\cdot, x(\cdot))}^{1}$. Also, by using Lemma 8 , we obtain $\forall n \geq 1$

$$
\left\|\mathcal{S}_{\alpha}(t-s) f_{n}(s)\right\| \leq|t-s|^{\alpha-1} M_{\mathcal{S}_{\alpha}} \vartheta(s) \in L^{1}\left(J, \mathbb{R}^{+}\right), \quad \forall t, s \in J, s \in(0, t] .
$$

Taking the limit as $n \rightarrow \infty$ on each sides (8), we get from the Lebesgue dominated convergence Theorem

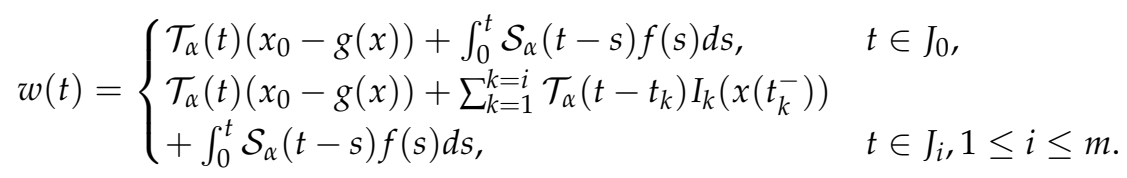

Thus, $w \in Q(x)$.

Let $x_{1}, x_{2} \in P C\left(J, \mathbb{R}^{n}\right)$, we show that $\exists \eta \in(0,1)$ such that

$$
H_{d}\left(Q\left(x_{1}\right), Q\left(x_{2}\right)\right) \leq \eta\left\|x_{1}-x_{2}\right\|_{P C}, \quad \forall x_{1}, x_{2} \in P C\left(J, \mathbb{R}^{n}\right) .
$$

Let $z_{1} \in Q\left(x_{1}\right)$, from (7) there is $f_{1} \in S_{F\left(\cdot, x_{1}(\cdot)\right)}^{1}$ with

$$
z_{1}(t)= \begin{cases}\mathcal{T}_{\alpha}(t)\left(x_{0}-g\left(x_{1}\right)\right)+\int_{0}^{t} \mathcal{S}_{\alpha}(t-s) f_{1}(s) d s, & t \in J_{0}, \\ \mathcal{T}_{\alpha}(t)\left(x_{0}-g\left(x_{1}\right)\right)+\sum_{k=1}^{k=i} \mathcal{T}_{\alpha}\left(t-t_{k}\right) I_{k}\left(x\left(t_{k}^{-}\right)\right) & \\ +\int_{0}^{t} \mathcal{S}_{\alpha}(t-s) f_{1}(s) d s, & t \in J_{i}, i=1, \cdots, m .\end{cases}
$$

From $\mathrm{H}_{3}(\mathrm{a})$, we get

$$
H_{d}\left(F\left(t, x_{1}\right), F\left(t, x_{2}\right)\right) \leq \vartheta(t)\left\|x_{1}(t)-x_{2}(t)\right\| .
$$

Then, $\exists \mu \in F\left(t, x_{2}(t)\right)$ with

$$
\left\|f_{1}(t)-\mu\right\| \leq \vartheta(t)\left\|x_{1}(t)-x_{2}(t)\right\|, \quad t \in J .
$$

Now, consider the map $\Lambda: J \rightarrow 2^{\mathbb{R}^{n}}$ defined as

$$
\Lambda(t)=\left\{\mu \in \mathbb{R}^{n}:\left\|f_{1}(t)-\mu\right\| \leq \vartheta(t)\left\|x_{1}(t)-x_{2}(t)\right\|\right\} .
$$

Since $f_{1}, \vartheta, x_{1}, x_{2}$ are measurable, then by using proposition 3.4 in [41], $\Lambda(t) \cap F\left(t, x_{2}(t)\right)$ is measurable. Moreover, its values are nonempty and compact. So, from Theorem 1.3.1 in [42], there exists $f_{2} \in F\left(t, x_{2}(t)\right)$ with

$$
\left\|f_{1}(t)-f_{2}(t)\right\| \leq \vartheta(t)\left\|x_{1}(t)-x_{2}(t)\right\|, \text { a.e. } t \in J .
$$

Let us define

$$
z_{2}(t)= \begin{cases}\mathcal{T}_{\alpha}(t)\left(x_{0}-g\left(x_{2}\right)\right)+\int_{0}^{t} \mathcal{S}_{\alpha}(t-s) f_{2}(s) d s, & t \in J_{0}, \\ \mathcal{T}_{\alpha}(t)\left(x_{0}-g\left(x_{2}\right)\right)+\sum_{k=1}^{k=i} \mathcal{T}_{\alpha}\left(t-t_{k}\right) I_{k}\left(x\left(t_{k}^{-}\right)\right) & \\ +\int_{0}^{t} \mathcal{S}_{\alpha}(t-s) f_{2}(s) d s, & t \in J_{i} .\end{cases}
$$


Obviously, $z_{2} \in Q\left(x_{2}\right)$ and if $t \in J_{0}$ we have

$$
\begin{gathered}
\left\|z_{1}(t)-z_{2}(t)\right\| \leq\left\|\mathcal{T}_{\alpha}(t)\right\|\left\|g\left(x_{1}\right)-g\left(x_{2}\right)\right\|+\int_{0}^{t}\left\|\mathcal{S}_{\alpha}(t-s)\right\|\left\|f_{1}(t)-f_{2}(t)\right\| d s \\
\leq\left[M_{\mathcal{T}_{\alpha}} N+M_{\mathcal{S}_{\alpha}} \frac{b^{\alpha}}{\alpha}\|\vartheta\|\right]\left\|x_{1}-x_{2}\right\|_{P C} .
\end{gathered}
$$

Similarly, when $t \in J_{i}, i=1, \cdots, m$, we have

$$
\left\|z_{1}(t)-z_{2}(t)\right\| \leq\left[M_{\mathcal{T}_{\alpha}}(N+\zeta)+M_{\mathcal{S}_{\alpha}} \frac{b^{\alpha}}{\alpha}\|\vartheta\|\right]\left\|x_{1}-x_{2}\right\|_{P C} .
$$

Consequently,

$$
H_{d}\left(Q\left(x_{1}\right), Q\left(x_{2}\right)\right) \leq \eta\left\|x_{1}-x_{2}\right\|_{P C} \forall x_{1}, x_{2} \in P C\left(J, \mathbb{R}^{n}\right),
$$

where, $\eta=\left[M_{\mathcal{T}_{\alpha}}(N+\zeta)+M_{\mathcal{S}_{\alpha}} \frac{b^{\alpha}}{\alpha}\|\vartheta\|\right]$. From (6) we have $\eta<1$. Hence, $Q$ is $\eta$ contraction. Applying Theorem $1, Q$ has a fixed point which is a mild solution for (1).

In the next Theorem, we will show that $Q$ has convex and compact values.

Theorem 3. Let $Q$ be the multivalued map which defined in (7). Then, under the assumptions $H_{1}-H_{5}$ of Theorem 2 , the set $Q(x)$ is convex and compact $\forall x \in P C\left(J, \mathbb{R}^{n}\right)$.

Proof. From Theorem 2, we know that $Q(x) \neq \varnothing$ for each $x \in P C\left(J, \mathbb{R}^{n}\right)$.

At the first, we prove that $Q(x)$ is convex for each $x \in P C\left(J, \mathbb{R}^{n}\right)$. Let $y_{1}, y_{2} \in Q(x)$, and $\lambda \in(0,1)$. If $t \in J_{0}$, then from (7) we obtain

$$
\lambda y_{1}(t)+(1-\lambda) y_{2}(t)=\mathcal{T}_{\alpha}(t)\left(x_{0}-g(x)\right)+\int_{0}^{t} \mathcal{S}_{\alpha}(t-s)\left[\lambda f_{1}(s)-(1-\lambda) f_{2}(s)\right] d s,
$$

where $f_{1}, f_{2} \in S_{F(\cdot, x(\cdot))}^{1}$. As $F$ has convex value, $S_{F(\cdot, x(\cdot))}^{1}$ is convex. Then, $\left[\lambda f_{1}(s)-(1-\right.$ ג) $\left.f_{2}(s)\right] \in S_{F(\cdot, x(\cdot))}^{1}$. Thus, $\lambda y_{1}(t)+(1-\lambda) y_{2}(t) \in Q(x)$. By the same way we can show that $\lambda y_{1}(t)+(1-\lambda) y_{2}(t) \in Q(x)$ for $t \in J_{i}, i=1, \cdots, m$, Hence, $Q(x)$ is convex for each $x \in P C\left(J, \mathbb{R}^{n}\right)$.

Now, we demonstrate $Q$ maps any bounded set into bounded set in $P C\left(J, \mathbb{R}^{n}\right)$.

Let $\mathcal{G}_{r}=\left\{x \in P C\left(J, \mathbb{R}^{n}\right):\|x\|_{P C\left(J, \mathbb{R}^{n}\right)} \leq r\right\}$, where $r$ is a positive number. Obviously, $\mathcal{G}_{r}$ is bounded set in $P C\left(J, \mathbb{R}^{n}\right)$. Let $x \in \mathcal{G}_{r}, y \in Q(x)$, then by using $H_{3}, H_{4}$ and Lemma 8 for $t \in J_{0}$, we obtain

$$
\begin{aligned}
\|y(t)\| & \leq\left\|\mathcal{T}_{\alpha}(t)\right\|\left\|x_{0}-g(x)\right\|+\left\|\int_{0}^{t} \mathcal{S}_{\alpha}(t-s) f(s) d s\right\| \\
& \leq\left\|\mathcal{T}_{\alpha}(t)\right\|\left(\left\|x_{0}\right\|+\sup _{x \in \mathcal{G}_{r}}\|g(x)\|\right)+\int_{0}^{b}\left\|\mathcal{S}_{\alpha}(t-s)\right\| \vartheta(s) d s \\
& \leq M_{\mathcal{T}_{\alpha}}\left(\left\|x_{0}\right\|+\sup _{x \in \mathcal{G}_{r}}\|g(x)\|\right)+\frac{b^{\alpha}}{\alpha} M_{\mathcal{S}_{\alpha}}\|\vartheta\| .
\end{aligned}
$$

Similarly, if $t \in J_{i}, i=1, \cdots, m$, from $H_{3}, H_{4}, H_{5}$ and Lemma 8 we get

$$
\|y(t)\| \leq M_{\mathcal{T}_{\alpha}}\left(\left\|x_{0}\right\|+\sup _{x \in \mathcal{G}_{r}}\|g(x)\|\right)+M_{\mathcal{T}_{\alpha}} \sum_{k=1}^{k=i} \sup _{x \in \mathcal{G}_{r}}\left\|I_{k}\left(x\left(t_{k}^{-}\right)\right)\right\|+\frac{b^{\alpha}}{\alpha} M_{\mathcal{S}_{\alpha}}\|\vartheta\| .
$$

This means $Q$ maps bounded sets into bounded sets. 
Next, we show that $Q$ maps bounded sets into equicontinuous sets in $P C\left(J, \mathbb{R}^{n}\right)$. Let $\mathcal{B}=Q\left(\mathcal{G}_{r}\right)$. We need to show that $\mathcal{B}_{\overline{T_{i}}}$ is equicontinuous for every $i=0,1, \cdots, m$, where

$$
\mathcal{B}_{\overline{J_{i}}}=\left\{y^{*} \in C\left(\overline{J_{i}}, \mathbb{R}^{n}\right): y^{*}(t)=y(t), t \in J_{i}, y^{*}\left(t_{i}\right)=y\left(t_{i}^{+}\right), y \in \mathcal{B}\right\} .
$$

Let $y \in \mathcal{B}$. Then there exists $x \in \mathcal{G}_{r}$ with $y \in Q(x)$. Form the definition of $Q$, there is $f \in S_{F(., x(.))}^{1}$ with

$$
y(t)= \begin{cases}\mathcal{T}_{\alpha}(t)\left(x_{0}-g(x)\right)+\int_{0}^{t} \mathcal{S}_{\alpha}(t-s) f(s) d s, & t \in J_{0}, \\ \mathcal{T}_{\alpha}(t)\left(x_{0}-g(x)\right)+\sum_{k=1}^{k=i} \mathcal{T}_{\alpha}\left(t-t_{k}\right) I_{k}\left(x\left(t_{k}^{-}\right)\right) & \\ +\int_{0}^{t} \mathcal{S}_{\alpha}(t-s) f(s) d s, & t \in J_{i}, 1 \leq i \leq m .\end{cases}
$$

We consider the following cases:

Case 1. When $i=0$, let $t, t+\tau \in J_{0}=\left[0, t_{1}\right]$, then

$$
\begin{gathered}
\left\|y^{*}(t+\tau)-y^{*}(t)\right\|=\|y(t+\tau)-y(t)\| \leq\left\|\mathcal{T}_{\alpha}(t+\tau)\left(x_{0}-g(x)\right)-\mathcal{T}_{\alpha}(t)\left(x_{0}-g(x)\right)\right\|+ \\
\left\|\int_{0}^{t+\tau} \mathcal{S}_{\alpha}(t+\tau-s) f(s) d s-\int_{0}^{t} \mathcal{S}_{\alpha}(t-s) f(s) d s\right\| \\
\leq Q_{1}+Q_{2}+Q_{3}
\end{gathered}
$$

where

$Q_{1}=\left\|\mathcal{T}_{\alpha}(t+\tau)\left(x_{0}-g(x)\right)-\mathcal{T}_{\alpha}(t)\left(x_{0}-g(x)\right)\right\|$,

$Q_{2}=\left\|\int_{0}^{t}\left[\mathcal{S}_{\alpha}(t+\tau-s)-\mathcal{S}_{\alpha}(t-s)\right] f(s) d s\right\|$,

$Q_{3}=\left\|\int_{t}^{t+\tau} \mathcal{S}_{\alpha}(t+\tau-s) f(s) d s\right\|$.

We will show that $Q_{i} \rightarrow 0$ as $\tau \rightarrow 0$ for $i=1,2,3$.

$$
\lim _{\tau \rightarrow 0} Q_{1} \leq\left(\left\|x_{0}\right\|+\sup _{x \in \mathcal{G}_{r}}\|g(x)\|\right) \lim _{\tau \rightarrow 0}\left\|\mathcal{T}_{\alpha}(t+\tau)-\mathcal{T}_{\alpha}(t)\right\|=0,
$$

independently of $x \in \mathcal{G}_{r}$.

For $Q_{2}$, from the definition of $\mathcal{S}_{\alpha}(t)$ and Lebesgue dominated convergence Theorem we get

$$
\begin{aligned}
\lim _{\tau \rightarrow 0} Q_{2} & \leq \lim _{\tau \rightarrow 0} \int_{0}^{t}\left\|\mathcal{S}_{\alpha}(t+\tau-s) f(s)-\mathcal{S}_{\alpha}(t-s) f(s)\right\| d s \\
& \leq \int_{0}^{t} \lim _{\tau \rightarrow 0}\left\|\mathcal{S}_{\alpha}(t+\tau-s) f(s)-\mathcal{S}_{\alpha}(t-s) f(s)\right\| d s=0,
\end{aligned}
$$

independently of $x \in \mathcal{G}_{r}$.

For $Q_{3}$,

$$
\lim _{\tau \rightarrow 0} Q_{3}=\lim _{\tau \rightarrow 0}\left\|\int_{t}^{t+\tau} \mathcal{S}_{\alpha}(t+\tau-s) f(s) d s\right\| \leq M_{\mathcal{S}_{\alpha}}\|\vartheta\| \lim _{\tau \rightarrow 0} \int_{t}^{t+\tau}(t-s)^{t+\tau-s} d s=0,
$$

independently of $x \in \mathcal{G}_{r}$.

Therefore,

$$
\lim _{\tau \rightarrow 0}\|y(t+\tau)-y(t)\|=0 .
$$


Case 2. When $t \in J_{i}, i \in\{1,2, \cdots, m\}$. Let $t, t+\tau \in J_{i}$, from (7) we have

$$
\begin{aligned}
\left\|y^{*}(t+\tau)-y^{*}(t)\right\|= & \|y(t+\tau)-y(t)\| \leq\left\|\mathcal{T}_{\alpha}(t+\tau)\left(x_{0}-g(x)\right)-\mathcal{T}_{\alpha}(t)\left(x_{0}-g(x)\right)\right\| \\
& +\sum_{k=1}^{k=i}\left\|\mathcal{T}_{\alpha}\left(t+\tau-t_{k}\right) I_{k}\left(x\left(t_{k}^{-}\right)\right)-\mathcal{T}_{\alpha}\left(t-t_{k}\right) I_{k}\left(x\left(t_{k}^{-}\right)\right)\right\| \\
& +\left\|\int_{0}^{t+\tau} \mathcal{S}_{\alpha}(t+\tau-s) f(s) d s-\int_{0}^{t} \mathcal{S}_{\alpha}(t-s) f(s) d s\right\| .
\end{aligned}
$$

Arguing as in the first case, we obtain

$$
\lim _{\tau \rightarrow 0}\|y(t+\tau)-y(t)\|=0
$$

Case 3. When $t=t_{i}, i=1,2, \cdots, m$. Let $\tau>0$ and $\delta>0$ such that $t_{i}+\tau \in J_{i}$ and $t_{i}<\delta<t_{i}+\tau \leq t_{i+1}$, then we have

$$
\left\|y^{*}\left(t_{i}+\tau\right)-y^{*}\left(t_{i}\right)\right\|=\lim _{\delta \rightarrow t_{i}^{+}}\left\|y\left(t_{i}+\tau\right)-y(\delta)\right\| .
$$

From (7), we obtain

$$
\begin{aligned}
\left\|y\left(t_{i}+\tau\right)-y(\delta)\right\| \leq & \left\|\mathcal{T}_{\alpha}\left(t_{i}+\tau\right)\left(x_{0}-g(x)\right)-\mathcal{T}_{\alpha}(\delta)\left(x_{0}-g(x)\right)\right\| \\
& +\sum_{k=1}^{k=i}\left\|\mathcal{T}_{\alpha}\left(t_{i}+\tau-t_{k}\right) I_{k}\left(x\left(t_{k}^{-}\right)\right)-\mathcal{T}_{\alpha}\left(\delta-t_{k}\right) I_{k}\left(x\left(t_{k}^{-}\right)\right)\right\| \\
& +\left\|\int_{0}^{t_{i}+\tau} \mathcal{S}_{\alpha}\left(t_{i}+\tau-s\right) f(s) d s-\int_{0}^{\delta} \mathcal{S}_{\alpha}(\delta-s) f(s) d s\right\| .
\end{aligned}
$$

Arguing as in the first case, we get

$$
\lim _{\substack{\tau \rightarrow 0 \\ \delta \rightarrow t_{i}^{+}}}\left\|y\left(t_{i}+\tau\right)-y(\delta)\right\|=0 .
$$

From (9)-(11), we conclude that $\mathcal{B}_{\overline{T_{i}}}$ is equicontinuous in $P C\left(J, \mathbb{R}^{n}\right)$ for each $i=0,1, \cdots, m$. From Azelà-Ascoli Theorem, $Q$ is completely continuous. Moreover, from Theorem 2 we have $Q(x)$ is closed. So, $Q(x)$ is compact for all $x \in P C\left(J, \mathbb{R}^{n}\right)$.

Let $\mathcal{E}_{x_{0}}([0, \gamma])$ be the set of all solution of $(1)$ on $[0, \gamma]$ where $0<\gamma \leq b$. From Theorem 2, we know that $\operatorname{Fix}(Q)=\mathcal{E}_{x_{0}}([0, \gamma]) \neq \varnothing$. In the following theorem we shall prove that $\mathcal{E}_{x_{0}}([0, \gamma])$ has an infinite dimension for any $x_{0}$.

Theorem 4. Let $F:[0, \gamma] \times \mathbb{R}^{n} \rightarrow P_{c k}\left(\mathbb{R}^{n}\right)$. Suppose that $H_{1}-H_{5}$ hold and $\chi\{t: \operatorname{dim} F(t, x)<$ 1 for some $\left.x \in \mathbb{R}^{n}\right\}=0$, where $\chi$ is the Lebesgue measure. Then, for all $\gamma, 0<\gamma<$ $\min \left\{\left(\frac{\left(1-M_{\mathcal{T}_{\alpha}}(N+\zeta)\right) \alpha}{M_{\mathcal{S}_{\alpha}}\|\vartheta\|}\right)^{\frac{1}{\alpha}}, b\right\}$, the set $\mathcal{E}_{x_{0}}([0, \gamma])$ has an infinite dimension for any $x_{0}$.

Proof. Consider the operator $Q: P C\left([0, \gamma], \mathbb{R}^{n}\right) \rightarrow P\left(P C\left([0, \gamma], \mathbb{R}^{n}\right)\right)$ defined by: for every $x \in P C\left([0, \gamma], \mathbb{R}^{n}\right) y \in Q(x)$ iff

$$
y(t)= \begin{cases}\mathcal{T}_{\alpha}(t)\left(x_{0}-g(x)\right)+\int_{0}^{t} \mathcal{S}_{\alpha}(t-s) f(s) d s, & t \in J_{0}, \\ \mathcal{T}_{\alpha}(t)\left(x_{0}-g(x)\right)+\sum_{k=1}^{k=i} \mathcal{T}_{\alpha}\left(t-t_{k}\right) I_{k}\left(x\left(t_{k}^{-}\right)\right) & \\ +\int_{0}^{t} \mathcal{S}_{\alpha}(t-s) f(s) d s, & t \in J_{i}, 1 \leq i \leq p,\end{cases}
$$

where $f \in S_{F(\cdot, x(\cdot))}^{1}$. From Theorem 2 we have $\operatorname{Fix}(Q)=\mathcal{E}_{x_{0}}([0, \gamma])$. So, we will prove that Fix $(Q)$ has an infinite dimension. From Theorem $3, Q(x)$ is convex and compact for all $x \in$ 
$\operatorname{PC}\left([0, \gamma], \mathbb{R}^{n}\right)$. Moreover, from Theorem $2 Q$ is contraction if $\left(M_{\mathcal{T}_{\alpha}}(N+\zeta)+M_{\mathcal{S}_{\alpha}} \frac{\gamma^{\alpha}}{\alpha}\|\vartheta\|\right)<$ 1 or $\gamma<\left(\frac{\left(1-M_{\mathcal{T}_{\alpha}}(N+\zeta)\right) \alpha}{M_{\mathcal{S}_{\alpha}}\|\vartheta\|}\right)^{\frac{1}{\alpha}}$. We will prove that $\operatorname{dim} Q(x) \geq q$ for any $x \in P C\left([0, \gamma], \mathbb{R}^{n}\right)$ and $q \in \mathbb{N}$. Put $R(t)=F(t, x(t))$. From Lemma 3, there are linearly independent measurable selections $f_{1}(\cdot), f_{2}(\cdot), \cdots, f_{q}(\cdot)$ of $R$. Let $y_{j}(t) \in Q(x), j=1, \cdots, q$, such that

$$
y_{j}(t)= \begin{cases}\mathcal{T}_{\alpha}(t)\left(x_{0}-g(x)\right)+\int_{0}^{t} \mathcal{S}_{\alpha}(t-s) f_{j}(s) d s, & t \in J_{0}, \\ \mathcal{T}_{\alpha}(t)\left(x_{0}-g(x)\right)+\sum_{k=1}^{k=i} \mathcal{T}_{\alpha}\left(t-t_{k}\right) I_{k}\left(x\left(t_{k}^{-}\right)\right) & \\ +\int_{0}^{t} \mathcal{S}_{\alpha}(t-s) f_{j}(s) d s, & t \in J_{i}, 1 \leq i \leq p .\end{cases}
$$

We suppose that $\sum_{j=1}^{q} a_{j} y_{j}(t)=0$, a.e. $t \in[0, \gamma]$. By taking Caputo derivatives a.e. in $[0, \gamma]$, we obtain $\sum_{j=1}^{q} a_{j} f_{j}(t)=0$, a.e. $t \in[0, \gamma]$. Since $f_{1}(\cdot), f_{2}(\cdot), \cdots, f_{q}(\cdot)$ are linearly independent, $a_{j}=0 \forall j$. So, $y_{j}(\cdot)$ are linearly independent. Hence, $Q(x)$ contains $q$-dimensional simplex. Therefore, $\operatorname{dim} Q(x) \geq q$. From Theorem 2 , we have $F i x(Q) \neq \varnothing$ and by Definition 2, we get $\operatorname{Fix}(Q) \subset Q(\operatorname{Fix}(Q))$. Then,

$$
\Omega(\operatorname{Fix}(Q)) \leq \Omega(Q(\operatorname{Fix}(Q))),
$$

where $\Omega$ is the Hausdorff measure of noncompactness. Thus, $Q$ is condensing ( see [43]). This implies that, Fix $(Q)$ is compact. Let $I$ be the identity operator, consider the map $I-Q: F i x(Q) \rightarrow P_{c k}\left(\mathbb{R}^{n}\right)$. Suppose that $\operatorname{dim} \operatorname{Fix}(Q)<n$, then by using Lemma 5, there is a continuous selection $\sigma$ of $I-Q$ with $\sigma(x) \neq 0$ for each $x \in \operatorname{Fix}(Q)$. So, there is a continuous selection $v$ of $F: \operatorname{Fix}(Q) \rightarrow P_{c k}\left(\mathbb{R}^{n}\right)$ with no fixed points. Now, define $\mathcal{F}: \mathbb{R}^{n} \rightarrow P_{c k}\left(\mathbb{R}^{n}\right)$ as

$$
\mathcal{F}(x)= \begin{cases}Q(x), & x \in \mathbb{R}^{n} \backslash \operatorname{Fix}(Q) \\ v(x), & x \in \operatorname{Fix}(Q)\end{cases}
$$

By using Lemma 6, $\mathcal{F}$ admits a continuous selection $f: \mathbb{R}^{n} \rightarrow \mathbb{R}^{n}$. Consequently, $f$ is a continuous selection of $Q$ such that $\operatorname{Fix}(f)=\varnothing$ and $v=f$ on $\operatorname{Fix}(Q)$, this contradicts Lemma 4. Therefore, Fix $(Q)=\mathcal{E}_{x_{0}}([0, \gamma])$ is infinite dimensional.

\section{Example}

Consider the following fractional impulsive differential inclusion as an application of our results.

$$
\begin{cases}{ }^{c} D_{t}^{\frac{1}{2}} x(t) \in x(t)+F(t, x(t)), & t \in J=[0,1], x \in \mathbb{R} \\ x\left(t_{i}^{+}\right)=x\left(t_{i}^{-}\right)+\frac{1}{2^{i}}, & i=1, \cdots, m, \\ x(0)=0, & \end{cases}
$$

where $A=1$.

Let $F:[0,1] \times \mathbb{R} \rightarrow P(\mathbb{R})$ such that

$$
F(t, x(t))=\left(\frac{t}{\sqrt{(t+1)}}, \frac{\cot ^{-1} x}{2 \sqrt{t^{2}+1}}+\frac{\sin t}{\sqrt{t+1}}\right) .
$$

Obviously,

$$
\|F(t, x)\| \leq \frac{\pi}{2 \sqrt{t^{2}+1}}+\frac{\sin t}{\sqrt{t+1}}=\vartheta(t) .
$$

For each $i=1, \cdots . m$, we define $I_{i}: \mathbb{R} \rightarrow \mathbb{R}$ as

$$
I_{i}\left(x\left(t_{i}\right)\right)=\frac{1}{2^{i}} .
$$


Clearly, $I_{i}$ satisfies $H_{5}$ for every $i=1, \cdots, m$.

Therefore, by Theorem 2 the system (12) has mild solution on $[0,1]$.

\section{Conclusions}

Nonlocal fractional impulsive differential inclusions involving a sectorial term are considered. The existence conditions are established under the situation when the multivalued function is Lipschitz. Further, we investigated the topological properties of the set of solutions. Our obtained results enhance and generalize many results that have been proven in recent papers.

Author Contributions: Conceptualization, N.A., K.G. and H.S.; Formal analysis, N.A., K.G., S.S. and H.S.; Funding acquisition, S.S.; Investigation, N.A., K.G.and H.S.; Methodology, N.A., K.G. and H.S.; Supervision, S.S.; Writing-review and editing, S.A. All authors have read and agreed to the published version of the manuscript.

Funding: This research received no external funding.

Institutional Review Board Statement: Not applicable.

Informed Consent Statement: Not applicable.

Data Availability Statement: Not applicable.

Conflicts of Interest: The authors declare no conflict of interest.

\section{References}

1. Das, S. Functional Fractional Calculus; Springer: Berlin, Germany, 2011.

2. Glocke, W.; Nonnemacher, T. A fractional calculus approach of self-similar protein dynamics. Biophys. J. 1995, 68, 46-53. [CrossRef]

3. Hilfer, R. Applications of Fractional Calculus in Physics; World Scientific: Singapore, 1999.

4. Kilbas, A.; Srivastava, H.; Trujillo, J. Theory and Applications of Fractional Differential Equations; North Holland Mathematics Studies; Elsevier Science, Publishers BV: Amesterdam, The Netherlands, 2006.

5. Miller, K.; Ross, B. An Introduction to the Fractional Calculus and Differential Equations; John Wiley: New York, NY, USA, 1993.

6. Oldham, K.; Spanier, J. The Fractional Calculus; Academic Press: New York, NY, USA, 1974.

7. Sabatier, J.; Agarwal, O.; Machado, J. Advances in Fractional Calculus; Theoretical Developments and Applications in Physics and Engineering; Springer: Berlin, Germany, 2007.

8. Zhou, Y. Basic Theory of Fractional Differential Equations; World Scientific: Singapore, 2014.

9. Agur, Z.; Cojocaru, L.; Mazaur, G.; Anderson, R.; Danon, Y. Pulse mass measles vaccination across age shorts. Proc. Natl. Acad. Sci. USA 1993, 90, 11698-11702. [CrossRef] [PubMed]

10. Ballinger, G.; Liu, X. Boundedness for impulsive delay differential equations and applications in populations growth models. Nonlinear Anal. 2003, 53, 1041-1062.

11. Lakshmikantham, V.; Bainov, D.; Simeonov, P. Theory of Impulsive Differential Equations; Modern Applied Mathematics; World Scientific: Teaneck, NJ, USA, 1989.

12. Onofrio, A. On pulse vaccination strategy in the SIR epidemic model with vertical transmission. Appl. Lett. 2005, 18, 729-732.

13. Samoilenko, A.; Perestyuk, N. Impulsive Differential Equations; World Scientific Series on Nonlinear Science, Series A: Monographs and Treatises 14; World Scientific Publishing: River Edge, NJ, USA, 1995.

14. Alsarori, N.; Ghadle, K. On the mild solution for nonlocal impulsive fractional semilinear differential inclusion in Banach spaces. J. Math. Model. 2018, 2, 239-258.

15. Alsarori, N.; Ghadle, K. Differential inclusion of fractional order with Impulse effects in Banach spaces. Nonlinear Funct. Anal. Appl. 2020, 1, 101-116.

16. Alsarori, N.; Ghadle, K. Nonlocal fractional differential inclusions with impulse effects and delay. J. Korean Soc. Ind. Appl. Math. 2020, 2, 229-242.

17. Bai, Z.; Dong, X.; Yin, C. Existence results for impulsive nonlinear fractional differential equation with mixed boundary conditions. Bound. Value Probl. 2016, 63, 2016. [CrossRef]

18. Gao, D.; Li, J. Existence results for impulsive fractional differential inclusions with two different caputo fractional derivatives. Discret. Dyn. Nat. Soc. 2019, 2019, 1-9. [CrossRef]

19. Ibrahim, A.; Alsarori, N. Mild solutions for nonlocal impulsive fractional semilinear differential inclusions with delay in Banach spaces. Appl. Math. 2013, 4, 40-56. [CrossRef]

20. Li, B.; Gou, H. Existence of solutions for impulsive fractional evolution equations with periodic boundary condition. Adv. Differ. Eq. 2017, 2017, 236. [CrossRef] 
21. Luo, Y. Existence for Semilinear Impulsive Differential Inclusions without Compactness. J. Dyn. Control. Syst. 2020, $26,663-672$. [CrossRef]

22. Mahmudov, M.; Unul, S. On existence of BVP's for impulsive fractional differential equations. Adv. Differ. Equ. 2017, $2017,15$.

23. Wang, J.; Feckan, M.; Zhou, Y. On the new concept of solutions and existence results for impulsive fractional evolutions. Dyn. PDE 2011, 4, 345-361.

24. Wang, J.; Feckan, M.; Zhou, Y. A survey on impulsive fractional differential equations. Fract. Calc. Appl. Anal. 2016, 19, 806-831. [CrossRef]

25. Agarwal, R.; Ahmad, B.; Alsaedi, A.; Shahzad, N. Existence and dimension of the set of mild solutions to semilinear fractional differential inclusions. Adv. Differ. Equ. 2012, 74, 1-10. [CrossRef]

26. Favini, A.; Yagi, A. Degenerate Differential Equations in Banach Spaces; Chapman and Hall/CRC Pure and Applied Mathematics: New York, NY, USA, 1998.

27. Ouahab, A. Fractional semilinear differential inclusions. Comput. Math. Appl. 2012, 64, 3235-3252. [CrossRef]

28. Kostić, M. Abstract Degenerate Volterra Integro-Differential Equations; Mathematical Institute SANU: Belgrade, Serbia, 2020.

29. Shu, X.; Lai, Y.; Chen, Y. The existence of mild solutions for impulsive fractional partial differential equations. Nonlinear Anal. 2011, 74, 2003-2011. [CrossRef]

30. Shu, X.; Shi, Y. A study on the mild solution of impulsive fractional evolution equations. Appl. Math. Comput. 2016, 273, 465-476. [CrossRef]

31. Wang, J.; Ibrahim, A.; Feckan, M. Nonlocal impulsive fractional differential inclusions with fractional sectorial operators on Banach spaces. Appl. Math. Comput. 2015, 257, 103-118. [CrossRef]

32. Wang, R.; Chen, D.; Xiao, T. Abstract fractional Cauchy problems with almost sectorial operators. J. Differ. Equ. 2012, 252, 202-235. [CrossRef]

33. Zhang, D.; Liang, Y. Existence and controllability of fractional evolution equation with sectorial operator and impulse. Adv. Differ. Equ. 2018, 2018, 219. [CrossRef]

34. Hu, S.; Papageorgiou, N. Handbook of Multivalued Analysis; Theory I. Kluwer: Dordrecht, The Netherlands, 1997.

35. Heinz, H. On the Behavior of measure of noncompactness with respect to differentiation and integration of vector-valued functions. Nonlinear Anal. 1973, 7, 1351-1371. [CrossRef]

36. Kamenskii, M.; Obukhowskii, V.; Zecca, P. Condensing Multivalued Maps and Semilinear Differential Inclusions in Banach Spaces; Walter de Gruyter: Berlin, Germany, 2001; Volume 7.

37. Dzedzej, Z.; Gelman, B. Dimension of the solution set for differential inclusions. Demonstr. Math. 1993, 26, 149-158. [CrossRef]

38. Saint-Raymond, J. Points fixes des multiapplications á valeurs convexes. (French) [Fixed points of multivalued maps with convex values]. C. R. Acad. Sci. Paris Sér. I Math. 1984, 298, 71-74.

39. Michael, E. Continuous selections. I. Ann. Math. 1956, 63, 361-382. [CrossRef]

40. Covitz, H.; Nadler, S. Multi-valued contraction mappings in generalized metric spaces. Isr. J. Math. 1970, 8, 5-11. [CrossRef]

41. Castaing, C.; Valadier, M. Convex Analysis and Measurable Multifunctions; Lecture Notes in Mathematics; Springer: Berlin, Germany, 1977.

42. Hiai, F.; Umegaki, H. Integrals, conditional expectation, and martingales of multivalued functions. J. Multivar. Anal. 1977, 7, 149-182. [CrossRef]

43. Akhmerov, R.; Kamenskii, M.; Potapov, A.; Rodkina, A.; Sadovskii, B. Measures of Noncompactness and Condensing Operators; Translated from the 1986 Russian original by A. Iacob. Operator Theory: Advances and applications; Birkhauser Verlag: Basel, Switzerland, 1992. 